\title{
COLLECTIONS ET MUSÉES D'ENTREPRISE : ENTRE PATRIMOINE ET STRATÉGIES DE COMMUNICATION Note CONCLUSIVE
}

\author{
Andrea CATEllani ${ }^{1}$ et Axel GryspeERDT ${ }^{2}$
}

Ce texte présente le contenu du dossier thématique sur les collections et la communication d'entreprise. Il retrace l'historique des musées d'entreprise et présente les différentes contributions du dossier, en terminant par quelques remarques globales et des pistes de travail.

Les musées d'entreprise tels qu'on les connaît aujourd'hui ont d'importants antécédents. L'initiative culturelle et artistique des entreprises est d'ailleurs loin d'être nouvelle. Si dans les années 1950-1960 se développe considérablement une série d'actions culturelles liées aux forces économiques en Europe comme aux États-Unis, jetant les bases d'une politique culturelle dans certaines grandes et moyennes entreprises, c'est parce que les fonde-

1 Andrea Catellani est professeur à la Faculté des Sciences économiques, sociales, politiques et de communication à l'Université catholique de Louvain, Louvain-laNeuve.

2 Axel Gryspeerdt est professeur émérite de la Faculté des Sciences économiques, sociales, politiques et de communication à l'Université catholique de Louvain, Louvain-la-Neuve et président de la fondation pour l'étude et le développement des collections d'art et de culture Collectiana, fondation d'utilité publique.

Recherches en communication, $\mathrm{n}^{\circ} 45$ - Article publié le 19/03/2019 
ments ont, en quelque sorte, déjà été établis dans les siècles précédents avec une efficacité certaine.

Un des mérites majeurs de l'article signé par François Mairesse est ainsi de montrer que les collections d'entreprises s'inscrivent dans une histoire plus longue que l'on ne l'imagine habituellement. François Mairesse nous aide en effet à mieux comprendre le passé en relevant l'impressionnant dispositif mis en place dès la fin du XVIII ${ }^{\mathrm{e}}$ siècle par les musées dits industriels et à partir du XIX ${ }^{\mathrm{e}}$ siècle par les musées qualifiés de commerciaux. Il montre surtout le rôle que ces deux types d'institutions ont joué dans le développement industriel et commercial des siècles qui ont précédé le nôtre. Il ne faut pas oublier que ces deux formes de musées se voulaient avoir de profonds effets à la fois sur la vitalité de l'économie dans son ensemble et sur la motivation des ouvriers et des artisans. Très vite, le commerce extérieur fut aussi un des objectifs visés par les musées de ces deux types, qui n'étaient pas sans liens avec les expositions internationales et universelles tendant à offrir en vitrine les meilleurs aspects des productions locales, régionales et surtout nationales.

Mairesse situe avec justesse et précision l'ensemble des fonctions remplies par ces musées et montre leur intrication profonde avec l'ensemble des rouages de l'économie. Il semble plus sceptique en ce qui concerne les rôles esthétique et scientifique de ces ancêtres des musées corporate et s'il évoque les questions de patrimoine et de mémoire collective, c'est pour mettre en évidence l'ambivalence des collections montrées, à la fois témoins du passé et mémoire des procédés industriels et outils toujours dépassés dans leurs aspects créatifs et technologiques. Mieux ne vaudrait-il pas exposer les productions industrielles et artisanales nouvelles, plutôt que celles du passé ? Cette interrogation et cette ambivalence restent d'application aujourd'hui et suscitent bien des débats autour d'entreprises de type technologique, comme par exemple celles qui produisent des calculatrices ou des ordinateurs. Toutefois les musées dont il nous parle n'hésitent pas non plus à mettre en évidence les procédures qui aboutissent à l'innovation. Et à la créativité. Mais ici aussi l'obsolescence guette. Que l'on pense par exemple à l'industrie de la parfumerie (voir à cet égard 
le texte de Paul Rasse et Vincent Lambert) ou à celle des colorants. Quant aux procédures qui permettent le développement commercial, elles semblent moins sujettes à être reléguées au rang des techniques passéistes. Dans l'ensemble, la fonction pédagogique de ces types de musées est certaine, mais elle se cantonne souvent au cadre économique étroit.

Par rapport à ces formes anciennes nées d'initiatives collectives, les musées d'entreprises contemporains ne se distingueraient-ils pas principalement par leur singularité ?

Un premier cas d'observation de pratiques singulières de collection d'entreprise contemporaine est judicieusement fourni par Anne Parizot qui s'attache à décortiquer les actions muséales mises au point par l'entreprise Michelin. Puisant dans les réserves de ses archives, Michelin tente depuis quelques années l'expérience muséale pour expliquer son parcours, baliser son « aventure » (terme utilisé par elle pour dénommer sa collection et le bâtiment qui l'accueille) et préfigurer que dans l'avenir elle continuera « avec efficacité » à aborder les questions de la mobilité par ses produits et par ses dérivés gastronomiques et cartographiques. Ainsi l'entreprise souhaite-t-elle prendre en compte le temps long qui caractérise la stratégie de ses dirigeants successifs. Misant sur ses symboliques, Michelin a réussi parallèlement à se constituer des groupes - voire même un public - de fidèles ou de fans, qui dépassent largement le milieu de ses employés - qu'elle implique surtout par son musée - en touchant sa clientèle directe (acheteurs des pneumatiques Michelin) et sa clientèle indirecte séduite par ses démarches publicitaires ou promotionnelles. $\mathrm{Si}$, de manière générale, la mise en collection est perçue comme un élément périphérique des entreprises au point tel qu'un changement de CEO peut la faire se dissoudre ou la reléguer du moins à l'arrière-plan, l'analyse d'Anne Parizot montre au contraire que certaines entreprises peuvent en faire un des dispositifs centraux de leur politique commerciale et de leur stratégie relationnelle avec ses divers publics.

Dans le processus de singularisation, les entreprises de la seconde moitié $\mathrm{du} \mathrm{Xx}^{\mathrm{e}}$ siècle et de notre siècle opèrent principale- 
ment en étant fières d'exposer le « meilleur » de leur production et de pouvoir recueillir des fruits par rapport à leurs concurrentes, qu'il s'agisse d'entreprises du même secteur ou d'entreprises qui œuvrent indirectement dans leur champ. À la panoplie des fonctions exercées, un certain nombre de musées d'entreprises contemporains n'hésitent pas à ajouter la mise en valeur des objets qu'ils exposent par une muséographie développée et à privilégier les éléments esthétiques. Qu'il s'agisse d'amélioration des espaces de travail ou encore de valorisation des objets les plus créatifs et de leurs « chefs-d'œuvres » artisanaux ou industriels.

Pour satisfaire à leurs fonctions de relations publiques, mais aussi de sauvetage des patrimoines esthétiques, les entreprises contemporaines ne se limitent plus à présenter leurs propres productions. Elles font entrer dans leur territoire des objets a priori fort éloignés de leurs sphères d'actions en organisant une collecte d'objets esthétiques liés aux beaux-arts. On pourra s'interroger quelque part sur le fait de comprendre s'il s'agit là prioritairement d'entreprises qui n'ont guère d'objets à présenter ou dont les objets sont très - trop - vite obsolètes, comme par exemple les institutions financières et bancaires et les secteurs technologiques (aviation, télécoms, informatique...), ou si le mouvement est davantage répandu. Rappelons à cet égard que, déjà dans les années 1960, certaines manufactures n'hésitaient pas à introduire des œuvres d'art dans les ateliers de production, pour stimuler la motivation et la créativité de ceux qui y travaillaient. Parmi elles, l'entreprise de tabac Peter Stuyvesant est souvent citée comme une figure pionnière.

Abordant successivement les opérations artistiques de la firme Louis Vuitton et les résidences d'artistes de l'entreprise Hermès, Eleni Mouratidou et Véronique Anderlini-Pillet s'intéressent aussi à des collections singulières de l'époque contemporaine. La contribution d'Eleni Mouratidou interroge, avec des outils sémio-pragmatiques, le dispositif muséal de Louis Vuitton (célèbre marque française du secteur du luxe, partie du groupe LVMH) du point de vue de son utilisation dans le cadre d'une stratégie de requalification. Les industries du luxe, selon cette auteure, 
se trouvent à devoir gérer la tension entre les effets symboliques potentiels de leur nature industrielle et l'axiologie du luxe, liée à des valeurs comme la rareté. Si les expositions d'acteurs comme Louis Vuitton sont des reconstructions historiques et valorisent un patrimoine, elles sont aussi des opérations de communication stratégique pour " maquiller la dimension industrielle du secteur ». La sémio-pragmatique permet d'observer dans les détails les modalités de cette gestion d'une tension axiologique. L'introduction de collections de mode de luxe dans des espaces à vocation artistique et l'utilisation de codes artistiques dans les espaces marchands, participent de cette gestion, qui produit des situations d'hybridation. La marque se donne à voir aussi comme « instance artistique ». Les stratégies de communication déployées sont alors liées à la catégorie de la « consommation ostentatoire ». L'auteure met donc en évidence la nature instrumentale de l'exposition et de la « captation » de l'art.

Véronique Anderlini-Pillet propose elle aussi une analyse concernant « l'artification » du luxe (notion reprise de Nathalie Heinich et Roberta Shapiro 2012), phénomène identifié comme lié à « l'économie de l'enrichissement » étudiée par Luc Boltanski et Arnaud Esquerre dans Enrichissement, une critique de la marchandise (2017). L'article propose une analyse du dispositif des « résidences d'artistes » dans le cadre de la fondation d'entreprise du groupe Hermès. Ces résidences participent d'un dispositif de patrimonialisation qui articule passé, présent et futur de la marque, et qui permet de construire une hybridation entre luxe et art. L'analyse se focalise sur différents aspects de ces résidences et de leur mise en discours et en signes, et les montre comme un exemple de reconfiguration de la collection d'entreprise pour l'adapter à un contexte de « nomadisme » et d'« hypermodernité ».

La contribution de Lise Renaud et Laure Marchis-Mouren analyse, elle aussi, les collections et les musées d'entreprise comme dispositifs destinés à articuler " culture et économie ", stratégies de patrimonialisation et de valorisation institutionnelle. Le cas observé est celui de quatre musées d'entreprises situées en 
Provence ; les auteures appliquent un regard inspiré par la sémiotique aux supports de communication externes et de visite experte, pour comprendre les « ressources symboliques de la patrimonialisation ». Le résultat de ce travail est l'identification de différentes formes de valorisation : une qui passe par la « figuration du passé »; une deuxième qui se fonde sur la « référence à une localité »; une troisième basée sur « la valeur de trouvaille à travers les figures du trésor »; enfin, une dernière liée à « l'évocation d'une mission désintéressée de transmission de la provençalité ». La patrimonialisation est donc analysée comme stratégie de communication capable, ici aussi, « d'édulcorer le rapport économique ».

En s'interrogeant sur les actions promotionnelles menées par les industries du parfum dans la région de Grasse, Paul Rasse et Vincent Lambert reviennent sur des questions d'actions collectives, telles qu'elles se vivent de nos jours. Rasse et Lambert relèvent les tensions vécues par les promoteurs d'initiatives industrielles et commerciales tendant à présenter les richesses et la vitalité de ces entreprises depuis la fin du XIX ${ }^{\mathrm{e}}$ siècle. Actions collectives, - notamment entreprises par un cluster parfum - par rapport aux actions singulières à volontés concurrentielles ? Présentation muséale des traditions ou au contraire des vecteurs du futur ? Prise en charge ou non des questions de responsabilité sociale et de développement durable ? La contribution des deux auteurs indique l'ensemble des paradoxes dans lequel sont confrontés les acteurs des initiatives muséales de la région, qu'ils soient privés ou publics. Ils attirent aussi les regards des lecteurs sur la diversité des solutions éventuelles, mais aussi sur les contraintes qui pèsent sur les organisateurs. Qu'il s'agisse de contraintes financières ou de contraintes d'images et de représentations du secteur.

Julie Deramond analyse avec des outils sémio-pragmatiques les espaces muséographiques ouverts par des " châteaux 》 vitivinicoles du Bordelais, interprétés comme résultats des stratégies de communication de l'industrie du vin. L'analyse inclut les objets exposés, les dispositifs de médiation in situ, mais aussi les dispositifs de communication comme les sites web. Cette analyse montre 
que ces musées du vin, chacun construit en lien avec l'identité du château correspondant, répondent à trois visées fondamentales (patrimoniale, touristique et économique), et participent à la lutte pour construire une valorisation culturelle du vin, pour le faire « monter en gamme » et augmenter sa valeur.

On doit à Roma Lambert d'épingler la toute grande diversité des pratiques ainsi que de l'ampleur des musées et des tailles des entreprises concernées, grandes, moyennes ou petites, aujourd'hui en France. La présentation d'accumulations artistiques ou d'objets liés au core business des entreprises n'est pas un phénomène isolé et la volonté communicationnelle des entreprises lancées dans une aventure artistique - musées, expositions, centres d'art, résidences d'artistes, vente d'objets liés au marketing culturel - est manifeste. Selon Roma Lambert, cette insertion dans la thématique artistique peut s'opérer de deux manières : soit les entreprises ont ellesmêmes un pied dans l'art de par leurs propres productions - « artisanat industriel » de luxe, affiches publicitaires réalisées par des artistes, etc. - soit elles ont acquis des œuvres d'art pour embellir leurs propres locaux ou pour stimuler l'image de leur marque. L'auteure réalise pincipalement un portrait des musées d'entreprises en France dans l'ensemble de leurs caractéristiques : secteur d'activité, motivation de départ, durabilité, choix de l'implantation et des lieux d'exposition, thématique(s), modalité des visites proposées, ce qui permet au lecteur d'avoir une vue panoramique sur la situation existante en France.

Il découle d'une lecture transversale des articles que les musées d'entreprises sont intimement liés aux opérations de communication, tantôt interne ou le plus souvent externe. Une de leurs motivations essentielle réside dans le fait qu'ils constituent un bon outil relationnel. Bien conçus - c'est-à-dire en cohérence avec celles-ci, ils composent un élément favorisant la culture d'entreprise et favorable à leur image. Certains d'entre eux misent en outre sur la convergence des collections avec leurs missions sociétales ou sociales. 
Ceci dit, certaines collections sont davantage liées à des choix stratégiques prononcés dans le but d'établir des liens avec leurs publics extérieurs, parmi lesquels leur clientèle est souvent citée, et/ou d'augmenter la motivation de leur personnel alors que d'autres collections paraissent davantage liées à des éléments d'ordre accidentel ou fortuit. Dans ces cas, un patron ou un membre du personnel passionné initie une collection qui sera ensuite " récupérée » par l'entreprise, trop heureuse et trop fière de bénéficier d'ensembles d'objets ou d'œuvres déjà constitués. Mais cette continuité n'apparaît souhaitable que si les ensembles en question convergent avec les autres éléments d'image recherchés par ces entreprises.

La fragilité des musées d'entreprise est plus prononcée que celle des musées institutionnels publics, bien que ces derniers soient de plus en plus souvent confrontés à des diminutions de subsides. À cet égard, il convient de noter que l'avenir des collections d'entreprise n'est guère abordée de front par la plupart des auteurs de ce dossier, bien que ceux-ci insistent régulièrement sur le fait que leur durabilité n'est jamais garantie. Le rôle joué par le CEO et par les conseils d'administration est considérable, même si certaines collections ont pu être entourées par des conseillers judicieux qui leur assurent une certaine stabilité. Les coûts de gestion, de conservation et d'acquisition restent énormes et les crises économiques et financières successives ont pesé sur les investissements. Quelques entreprises ont dès lors décidé d'évaluer les coûts comparés entre plusieurs actions de relations publiques, de marketing ou de communication interne. Ainsi la gestion d'une collection ou d'un centre d'art peut-elle s'avérer un investissement rentable ou du moins profitable par rapport à la réalisation d'autres types d'événements, par exemple sportifs ou d'investissements de nature publicitaire. Dans la volonté de s'assurer davantage de pérennité, certains conservateurs de musées d'entreprise n'hésitent pas à rechercher des partenariats, ce qui implique en même temps une ouverture plus grande vers l'extérieur et des collaborations multiples. Leur communication avec leurs différents publics en est d'autant plus bénéfique. 
Comme le soulignent Roma Lambert, Paul Rasse et Vincent Lambert, les responsables de ces collections doivent néamoins être attentifs à ne pas faire apparaître une ambition ou une motivation trop commerciale dans leur action. Ils risquent dans ce cas que les publics se désintéressent des pièces de collection qui leur sont montrées. Dans leur ensemble, ces différents auteurs plaident d'ailleurs pour une plus grande professionalisation des musées d'entreprise et pour une réflexion plus solide sur les modalités de leur fonctionnement, notamment en ce qui concerne leur accessibilité par les publics et la responsabilité qui est la leur à cet égard. Dans quelle mesure par exemple peut-on parler d'opérations d'éducation permanente réalisées par ces collections permettant aux visiteurs et aux membres des entreprises concernées de faire des découvertes et d'étoffer leurs connaissances (sur les objets présentés et aussi sur les valeurs promues par les entreprises)?

Il s'agit là d'une des questions qui restent largement ouvertes par ce dossier. Celui-ci n'évoquant encore que trop partiellement la manière dont les collections d'aujourd'hui s'ouvrent à la pluralité, à l'internationalisation - la plupart des collections analysées restent ancrées dans un seul territoire, alors que les entreprises se sont ouvertes à la scène internationale - à la numérisation et à de nouveaux publics.

Déjà un regard en dehors de la «francophonie » et de la France se justifierait pleinement : il permettrait de voir comment le musée d'entreprise apparaît comme dispositif de valorisation hybride dans d'autres contextes culturels et nationaux (nous pensons par exemple aux cas de Mercedes en Allemagne et de Ferrari en Italie, pour rester dans le domaine de l'automobile).

Ainsi le présent dossier fournit-il essentiellement un éclairage neuf et documenté sur une situation qui reste souvent méconnue et peu analysée jusqu'ici. Il s'agissait essentiellement par là d'élaborer des premiers jalons pour une approche plus systématique des situations et des problèmes vécus par les collections d'entreprise dans leur grande diversité de création, de créativité et d'animation. Il ouvre la voie à une réflexion plus profonde sur le rôle des objets dans notre société contemporaine et sur les diverses interprétations que l'on peut avoir de ceux-ci ainsi que sur les récits qui 
les accompagnent et sur les sauvegardes des traces du passé qu'ils réalisent. Qu'en cela les différents auteurs de ce numéro et ceux qui les ont soutenus soient vivement remerciés.

\section{Références}

Boltanski, Luc \& Esquerre, Arnaud (2017). Enrichissement. Une critique de la marchandise, Paris : Gallimard.

Heinich, Nathalie \& Shapiro Roberta (eds.) (2012). De l'artification. Enquête sur le passage à l'art. Paris : Éditions EHESS.

\section{(ब) $\Theta$ \\ BY NC ND Publié sous la licence Creative Commons}

«Attribution - Pas d'Utilisation Commerciale - Pas de Modification 4.0 International» (CC BY-NC-ND) 